A N N A L E S Annales de Bretagne et des Pays de l'Ouest

Anjou. Maine. Poitou-Charente. Touraine

116-1 | 2009

Le fait divers en province

\title{
Le fait divers en province
}

Présentation

Frédéric Chauvaud

\section{(2) OpenEdition}

Journals

Édition électronique

URL : http://journals.openedition.org/abpo/135

DOI : $10.4000 /$ abpo. 135

ISBN : 978-2-7535-1513-0

ISSN : 2108-6443

Éditeur

Presses universitaires de Rennes

Édition imprimée

Date de publication : 1 mars 2009

Pagination : $7-12$

ISBN : 978-2-7535-0875-0

ISSN : 0399-0826

\section{Référence électronique}

Frédéric Chauvaud, "Le fait divers en province », Annales de Bretagne et des Pays de l'Ouest [En ligne], 116-1 | 2009, mis en ligne le 31 décembre 2009, consulté le 19 avril 2019. URL : http:// journals.openedition.org/abpo/135; DOI : 10.4000/abpo.135 


\title{
Présentation \\ Le fait divers en province
}

\author{
Frédéric CHAUVAUD \\ Professeur d'histoire contemporaine, \\ université de Poitiers, \\ responsable de l'équipe «Sociétés conflictuelles » \\ du GERHICO-CERHILIM
}

Le fait divers a fait l'objet d'une unique exposition ${ }^{1}$ mais de nombreux travaux pluridisciplinaires de qualité ${ }^{2}$. La plupart, toutefois, étudient surtout la Belle Époque, en privilégiant quelques journaux nationaux. Le présent dossier s'attache tout d'abord à souligner que l'un des véritables tournants de I'histoire du fait divers date probablement de 1887. L'affaire Pranzini, oubliée aujourd'hui, se trouve pourtant placée au cœur de l'imaginaire de toute la société française «fin de siècle ». Vingt ans après l'affaire Troppmann, étudiée par Michelle Perrot ${ }^{3}$, elle envahit la Une des quotidiens, prolifère dans les pages intérieures, et s'établit à demeure pendant de longs mois. Mais, au-delà de quelques grandes affaires nationales retentissantes, le fait divers est aussi «provincial ${ }^{4}$ ». Sa perception comme son traitement et sa réception

1. Monestier, Alain, et Cheyonnaud, Jacques, Le Fait divers. Catalogue de l'exposition tenue au Musée des arts et traditions populaires à Paris, du 19 novembre 1982 au 18 avril 1983, Paris, Éditions de la Réunion des musées nationaux, 1982.

2. La bibliographie est aujourd'hui très riche; citons toutefois : PERROT, Michelle « Faitdivers et histoire au XIX ${ }^{\mathrm{e}}$ siècle », Annales ESC, $\mathrm{n}^{\circ}$ 4, juillet-août 1983, p. 911-919, et " Le fait divers : quelle histoire? » Digraphe, $\mathrm{n}^{\circ}$ 50, décembre 1989, p. 107-118; KaLIFA, Dominique, L'Encre et le sang : récits de crimes et société à la Belle Époque, Paris, Fayard, 1995, 351 p.; M'SILI, Marine, Le Faits divers en République. Histoire sociale de 1870 à nos jours, Paris, Éditions du CNRS, 2000, 311 p.; Ambroise-Rendu, Anne Claude, Petits Récits des désordres. Les faits divers dans la presse française au début de la III République à la Grande Guerre, Paris, Seli Arslan, 2004, 332 p.; DuBIED, Annick, Les Dits et les scènes du fait divers, Genève, Droz, 2004, $356 \mathrm{p}$.

3. Perrot, Michelle, «L'affaire Troppmann (1869) », L'Histoire, n 30 janvier 1981, p. 2837, repris dans Les Ombres de l'histoire, Paris, Flammarion, 2001, p. 283-298.

4. Voir par exemple, le récit-témoignage de BELLERET, Robert, qui fut notamment journaliste à Lyon au Progrès, Faits divers, Paris, Sabine Wespieser éditeur, 2007, 290 p. Voir aussi Kalifa, Dominique, "L'« insécurité » vue d'ailleurs. Courriers de I'Oise », dans Crime et culture au XIX ${ }^{\mathrm{e}}$ siècle, Paris, Perrin, 2005, p. 298-314. 
nécessitent d'en rendre compte en multipliant les approches ${ }^{5}$. Nul doute $q^{\prime}{ }^{\prime i l}$ est à la fois un objet de recherche complexe ${ }^{6}$, le portrait d'une époque ${ }^{7}$ et parfois le reflet des peurs des sociétés locales et régionales.

Le fait divers a colonisé, au XIX ${ }^{e}$ siècle, les colonnes des journaux et depuis, il ne les a pratiquement pas quittées. Relégué à la marge, au début du siècle, il a progressivement conquis droit de cité, abandonnant les canards sanglants ${ }^{8}$ pour s'imposer à la Une des grands quotidiens parisiens. Le Grand Dictionnaire universel du XIX ${ }^{e}$ siècle de Pierre Larousse en atteste. L'œuvre lexicographique la plus ample du XIX ${ }^{\mathrm{e}}$ siècle est aussi une œuvre engagée qui entend promouvoir l'idée républicaine, même si les premiers volumes sont publiés à la fin du Second Empire. Les auteurs du dictionnaire s'attachent à souligner la rencontre de l'opinion publique et du fait divers. L'opinion publique est d'abord définie comme un « sentiment universel ». Plus loin, selon le mode d'organisation de chaque notice, un collage de citations doit permettre de préciser les contours de la notion, de présenter ses multiples facettes, voire ses aspects contradictoires. Sont convoqués Necker, Benjamin Constant, Etienne et quelques autres. De la sorte, pour le lecteur, nul doute que l'opinion publique est bien « une puissance invisible qui commande jusque dans les palais du roi ». Le monument de Pierre Larousse offre aussi à la curiosité des lecteurs une partie encyclopédique et prend le risque d'historiciser le phénomène. En effet, "l'opinion publique naquit en France au milieu des orages de la révolution ». Mais, c'est "sous la seconde Restauration [que] commence le véritable règne de l'opinion publique. C'est une puissance qui s'élève, qui s'affermit et qui ne relève que d'elle-même. Bien plus, elle interroge les vieux pouvoirs, les somme de produire leurs titres et s'arroge le droit de les contrôler ${ }^{9}$ ". À l'article Faits divers, le rédacteur note: "Sous cette rubrique, les journaux groupent avec art et publient régulièrement les nouvelles de toute sorte qui courent le monde [...]. Le rédacteur chargé dans chaque journal de ce qu'il est convenu d'appeler la cuisine doit apporter une attention toute particulière dans le choix et la confection du fait divers ${ }^{10}$. " Le XIX siècle se caractérise aussi par la rencontre de l'opinion publique et du fait divers porté par une presse à large diffusion. En effet, la naissance d'une presse à grand tirage, dont les principales étapes sont aujourd'hui bien connues,

5. Merleau-Ponty, Maurice, "Sur les faits divers », Signes, Paris, Gallimard, p. 388-390 ; AuCLAIR, Georges, "Fait divers et pensée naïve », Critique, n 197, oct. 1963, p. 893-906; BARTHES, Roland, «Structure du fait divers », Essais, critiques, Paris Seuil, 1964, p. 188-197.

6. DUBIED, Annick, «Invasion péritextuelle et contaminations médiatiques. Le fait divers, une catégorie complexe ancrée dans le champ journalistique », Semen. Genres de la presse écrite et analyse du discours, 2001, $n^{\circ} 13$, p. 49-64.

7. Voir par exemple, Faits divers. Portraits de notre époque, collectif Paris-Obs, Paris, Les Petits Matins, 2005, $153 \mathrm{p}$.

8. Seguin, Jean-Pierre, Canards du XIX ${ }^{\mathrm{e}}$ siècle, Nouvelles à sensation, Paris, Armand Colin, coll. « Kiosque », 1959, $227 \mathrm{p}$.

9. LAROUSSE, Pierre, Grand Dictionnaire universel du XIX ${ }^{e}$ siècle, tome onzième, Paris, 1874, p. 1386.

10. Ibidem, tome huitième, 1872, p. 58. 
et l'élargissement considérable du lectorat sont des éléments essentiels. "L'âge d'or du fait divers " se situe bien au cours de ce siècle qui voit le passage des "canards » à la presse populaire ${ }^{11}$. Les conditions d'impression, l'accroissement de la qualité, l'augmentation du nombre de pages, les lois sur la presse et la censure, le recul de l'analphabétisme, les conditions de vie, l'accès des femmes aux livres et aux journaux... tout concourt à donner au fait divers une place inusitée. On le sait, ce dernier est une construction. Il faut en effet savoir " arranger et pimenter » un fait divers. Si les thèmes et leur traitement semblent presque constituer des invariants, du moins si l'on suit les analyses de Maurice Lever et de Jean Pierre Seguin, il n'en reste pas moins que ce qui change à partir de la monarchie de Juillet, c'est la prolifération du récit criminel dans la " boîte à journal », comme d'ailleurs dans la production romanesque. Désormais, il faut savoir raconter un assassinat, décrire une exécution, rapporter les dernières paroles d'un condamné. L'auteur de "fait-divers» doit être capable de faire partager ses émotions, de faire frémir son lecteur de la même manière que la foule qui a assisté au supplice du condamné à mort et a suivi avec avidité toutes les étapes de l'exécution capitale. Reste que le fait divers possède toujours un statut ambivalent : à la fois doté d'un pouvoir d'attraction magnétique, il provoque, de façon presque simultanée, l'ironie et le mépris.

Quelques grandes affaires ont rythmé le $x_{1 x}{ }^{e}$ siècle qui, en la matière, commence sans doute au début de la Restauration. En 1817, I'affaire Fualdès fait partie des "crimes d'autrefois ${ }^{12}$ ", de ces " affaires extraordinaires » qui transforment un crime ou un procès en fait divers, puis en fait de société. Par la suite, nombre de procès viennent prendre place, dont l'affaire Lafarge ${ }^{13}$ ou l'affaire Lacenaire ${ }^{14}$. Les canardiers et autres chroniqueurs judiciaires se contentent de recueillir des éléments divers, de les habiller un peu et de les présenter aux lecteurs. Si Fualdès est une victime et n'est guère représentée visuellement, en revanche des portraits, des dessins au trait, des photographies de criminels sont régulièrement offerts à la curiosité publique. Le personnage du criminel devient figure de la société contemporaine, héros de fait divers et de complaintes. Toutefois, il faudrait assurément distinguer plusieurs genres littéraires ou catégories d'écritures. Il existe par exemple depuis 1825 une littérature de cours d'assises, qui s'apparente à la relation de procès, comprenant parfois la transcription intégrale de l'acte d'accusation, de l'interrogatoire, des auditions des témoins, des questions posées à l'audience, des réquisitoires et des plaidoiries. Le plus souvent, les débats ne sont pas présentés dans leur intégralité, mais retranscrits, coupés, mis en scène. Les chroniqueurs de la

11. Lever, Maurice, Canards sanglants, naissance du fait divers, Paris, Fayard, 1993, $517 \mathrm{p}$.

12. Selon l'expression de Bouchardon, Pierre, Crimes d'autrefois, Paris, Perrin, 1926, $267 \mathrm{p}$.

13. Sur I'affaire Lafarge, voir notamment ADLER, Laure, L'Amour à I'arsenic: histoire de Marie Lafarge, Paris, Denoël, 1986, 219 p.

14. Demartinl, Anne Emmanuelle, L'Affaire Lacenaire, Paris, Aubier, 2001, 430 p. 
Gazette des tribunaux, le plus célèbre des périodiques judiciaires, possèdent une parfaite maîtrise du procédé, restituant de temps à autre l'ambiance du procès, l'atmosphère de la salle, les portraits des uns et des autres. II arrive aussi qu'un journaliste ou un correspondant aille encore plus loin et se fasse l'interprète de l'opinion commune dont il décrit les réactions stéréotypées et les émotions. Mais, en général, le fait divers se situe avant le procès qui, dans certains journaux, sert davantage de prétexte à revenir sur l'affaire elle-même ou à réactiver la mémoire du crime, de sa découverte, de ses circonstances, de ses protagonistes, de ses caractéristiques singulières ou universelles, qu'à donner véritablement un compte rendu précis et fidèle de l'audience des assises ou du tribunal correctionnel. II importe donc de s'attacher à la fois aux conditions de production du fait divers, aux événements énoncés, à leurs transcriptions, par le biais de l'écrit, et au sens qu'on peut leur donner ${ }^{15}$.

Dans les années trente du $x x^{e}$ siècle, Pierre Jacomet est, à sa manière, un des précurseurs de l'étude des faits divers. Certes, il s'attache davantage aux procès connus ou historiques, mais il donne matière à une première réflexion d'ensemble. Avocat à la cour de Paris et professeur à l'École des hautes études sociales, il tente notamment de cerner les relations entre la presse et l'opinion publique. Selon lui, cette dernière " est justicière. Aussi, suit-elle, avec plus de passion que jamais les débats judiciaires. Si le régime démocratique exacerbe les convoitises politiques, il rend aussi I'opinion publique plus attentive et plus vigilante [...]. Aussi, I'histoire de la Troisième République est-elle liée aux annales judiciaires par une nécessité interne, et si les procès ont jeté de vives lueurs sur la vie privée de la cité [...] elle [l'opinion publique] projette une lumière non moins crue sur la Troisième République ${ }^{16}$ ».

Le présent dossier, à la suite d'autres travaux, s'ouvre par l'examen d'un fait divers parisien, l'affaire Pranzini, qui ne peut cependant se cantonner à I'histoire de Paris. Certes Balzac utilise l'expression «faits-Paris » présentée comme l'ancêtre lexicographique du fait divers et Louis Chevalier considère que «le spectacle des faits divers est installé en permanence au beau milieu de l'histoire parisienne; il se donne comme le spectacle la cour des miracles ou comme la procession du pape des fous ${ }^{17}$ ". L'affaire Pranzini, trop souvent méconnue, voire ignorée, est pourtant un fait divers majeur qui illustre un tournant essentiel dans l'histoire des médias et des institutions judiciaires et policières. Son étude permet ainsi de mieux comprendre comment se fabrique un fait divers emblématique, de saisir ses logiques,

15. Ces deux aspects constituent quelques-unes des problématiques énoncées par AUCLAIR, Georges, Le Mana quotidien. Structures et fonctions de la chronique des faits divers, Paris, éditions Anthropos, 1970, 276 p.

16. JaCOMET, Pierre, Les Drames judiciaires du XIX siècle, Paris, Payot, 1929, p. 187-190. L'auteur précise encore que "N'y a-t-il pas, de notre temps, une autre source d'informations, qui est bien précieuse, la presse, c'est-à-dire ces feuilles qui racontent tout et qui sont le kaléidoscope où l'on voit se dérouler les événements du jour », p. 22.

17. Chevalier, Louis, Splendeurs et misères du fait divers, Paris, Perrin, 2004, p. 19. 
de suivre ses effets. Elle se situe en effet après le crime de Pantin qui voit le Petit Journal gagner un nombre considérable de lecteurs et après la loi du 29 juillet 1881 sur la presse.

Les contributions rassemblées dans la présente livraison des Annales de Bretagne et des Pays de l'Ouest s'attachent tout d'abord à cerner les contours du fait divers qui peut tantôt être enfermé dans une définition très précise ${ }^{18}$, tantôt échapper à toute taxinomie rigide, tant il est vrai qu'il est beaucoup plus varié que le seul récit criminel. André Gide avait dirigé une collection "Ne jugez pas », chez Gallimard, et il écrivait dans la deuxième livraison, publiée en 1930 : «Faits-Divers. Sans doute ils pourraient être plus suggestifs : nombre d'entre eux le seraient assurément si seulement l'on pouvait les regarder d'assez près ${ }^{19}$. » Aussi, l'ambition de la première partie consiste à proposer, à partir de sources poitevines, une sorte d'inventaire, soulignant toute la diversité du fait divers, de ses supports, comme de ses "lectures ». La visée de la seconde partie consiste à étudier la place des femmes dans les faits divers, renouvelant ainsi les approches usuelles. Elle s'ouvre par une étude privilégiant La Gazette des tribunaux, périodique judiciaire le plus célèbre du $\mathrm{XIX}^{\mathrm{e}}$ siècle et se poursuit par des études de cas, multipliant les éclairages régionaux à partir de trois villes: Rennes, Bordeaux et Poitiers. L'auteur du très célèbre Classes laborieuses, classes dangereuses faisait remarquer, dans son cours au Collège de France, que toute étude d'ensemble devrait d'abord s'intéresser à " l'immédiat du fait divers » puis aux « effets de chocs » qu'il provoque chez le lecteur. II insistait ensuite sur les difficultés de "l'entreprise d'histoire » qui ne peut rivaliser avec le récit romanesque. Reste alors à se demander s'il n'est pas envisageable d'aller plus loin. Sans doute peut-on distinguer une première acception du fait divers qui se rapporte à ce qui arrive : "Un incident qui, dans l'état initial, en soi, n'est pas même, et surtout pas, un événement. Une chose à certains égards insignifiante, mais dont on peut penser aussi qu'elle est pleine de signification. "Ce fait divers n'intéresse que les acteurs directs concernés par lui, mais il existe aussi un second temps: "Ensuite intervient le choix par la presse [...] de cette virtualité pour la transformer en fait divers réel, ou du moins pour l'inscrire à la page des faits divers ${ }^{20}$. " Les articles rassemblés ci-dessous répondent à ces interrogations. En effet, ils ne se contentent pas du seul niveau des énoncés. Ils ambitionnent de caractériser le fait divers dans tous ses états, puis de montrer que, au-delà des effets de répétition, il est bien le révélateur d'une société à un moment donné, de ses relations sociales, de ses angoisses comme de ses aspirations.

18. FERRO, Marc, « Faits divers, faits d'histoire », Annales ESC, 1983, n 4, p. 821-825.

19. GIDE, André, L'Affaire Redureau, suivie de Faits Divers, Paris, Gallimard, 1930, p. 104.

20. Chevalier, Louis, Splendeurs..., op. cit., p. 25-26. 
RÉSUMÉ

Le fait divers a colonisé l'imaginaire de la société et fait la conquête des colonnes des journaux et depuis, il ne les a pratiquement pas quittées. La présente livraison des ABPO entend tout d'abord s'attacher à la diversité des faits divers, qui malgré des travaux de qualité et des définitions diverses, ne se laissent pas enfermer dans une acception trop rigide. Pour en saisir toute l'importance, le numéro s'ouvre par l'analyse du fait divers le plus célèbre de la seconde moitié du xix ${ }^{e}$ siècle, se poursuit par l'étude des faits divers régionaux, en multipliant les angles d'approche, puis se referme par l'analyse de la place des femmes dans les faits divers mis en scène par la presse des départements ou par La Gazette des tribunaux. De la sorte, les articles rassemblés ici ont pour visée de caractériser le fait divers tout en soulignant qu'il constitue une voie d'accès privilégié pour comprendre les aspirations et les angoisses d'une société à un moment donné.

\section{ABSTRACT}

The "fait divers" sank into the society imagination and entered the newspapers columns, and since, it pratically did not leave them. This edition deals firstly with the diversity of "faits divers", that in spite of worthy researches and various definitions haven't been confine in a too rigid meaning. To seize all the importance of it, this edition begins with the analysis of the most second half of $19^{\mathrm{h}}$ century's famous "fait divers". Then it continues with the study of different regional "faits divers", by multiplying the ways to approach it. Then this edition is ended with the analysis of the place of women in the "faits divers", related by the departemental press or by La Gazette des tribunaux. To sum up, these articles gathered in this edition have as an aiming to characterize the "faits divers" while underlining that it constitutes a privilegiate means to understand the aspirations and angsts of a certain society at a given time. 